\title{
Treue zu sich selbst
}

\section{François Jacobs Autobiographie «La Statue Intérieure»}

Die Molekularbiologie ist keine 40 Jahre alt und schon Geschichte. Die wunderbare Struktur der Nukleinsäure, der Nachrichtenfluß vom Zellkern zum Zelleib, die Geheimschrift, in der die Baupläne des Lebens niedergelegt sind, alle diese Bestandteile eines der großen Erkenntnisabenteuer unseres Jahrhunderts entlocken heute dem Mittelschüler höchstens ein Gähnen. Doch für alle, die aus der Nähe oder von fern Zeugen dieser Revolution waren, bleibt ihre Frühzeit, als im allmählich sich aufhellenden Halbdunkel getappt wurde, ein Drama größter Intensität. Mehrere der unmittelbar Beteiligten haben jene aufregenden Jahre geschildert, und Wissenschaftshistoriker haben sich bemüht, die verschiedenen Stränge zu entwirren, die zu den entscheidenden Entdeckungen geführt haben: Eine der Schlüsselfiguren legt nun ihre Autobiographie vor ${ }^{1}$.

François Jacob, Sproß einer jüdischen französischen Familie, erzählt sein Leben von den Anfängen bis zum wissenschaftlichen Durchbruch. Dieses Leben beginnt als das zärtlich geliebte Einzelkind einer zärtlich geliebten Mutter. Dem hochbegabten, empfindsamen Jungen hätte man die reibungslose Laufbahn des französischen Intellektuellen voraussagen dürfen, wäre da nicht eine schreckliche Zäsur eingetreten: nach kaum begonnenem Medizinstudium treffen den Zwanzigjährigen im Juni 1940 der Tod der Mutter und der Zusammenbruch Frankreichs. Beide Ereignisse verschmelzen ihm zu einem unersetzlichen Verlust. Mit Resten polnischer Divisionen schifft er sich nach England ein und schließt sich mit den Allerersten den Freien Franzosen de Gaulles an.

Damit beginnt ein zweiter Lebensabschnitt, in dem der junge Mann, der gewiß nichts von einem Haudegen hat, sich und der Welt beweisen will, daß er, «passionnément français», jedes Opfer für seine Heimat zu bringen bereit ist. Obschon er sich zur Artillerie meldet (Vater und Großvater hatten in der Artillerie gedient), wird er der Sanität zugeteilt. Er macht den Afrikafeldzug unter Leclerc mit, landet mit der 2. Panzerdivision als Teil der 3. Amerikanischen Armee in der Normandie und wird schwer verwundet, so daß er weder den Taumel des Einmarsches in Paris noch den Waffenstillstandstag miterleben kann.

1 François Jacob, La statue intérieure. Paris, Ed. O. Jacob, Seuil, 1987. 


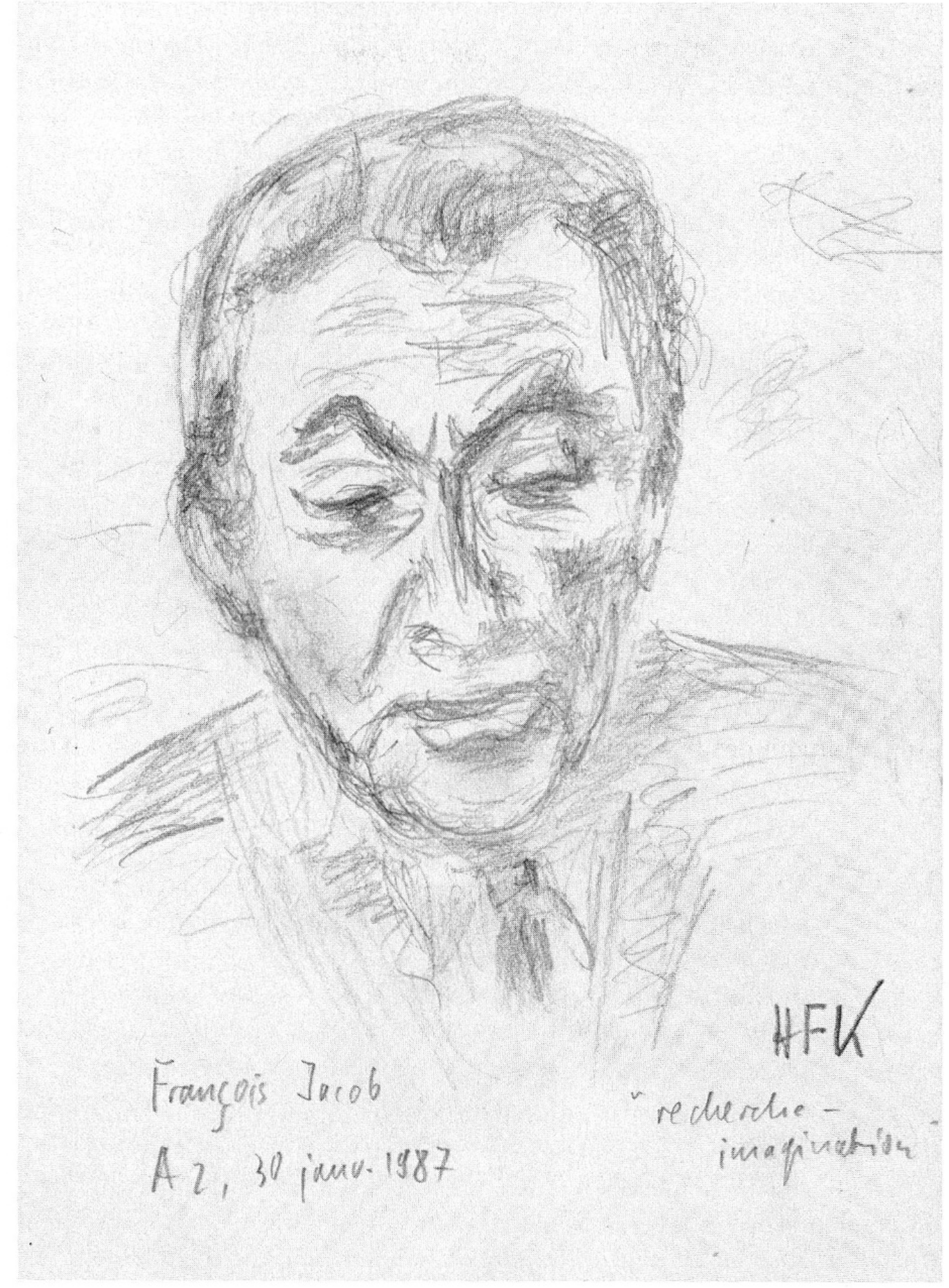

François Jacob. Am französischen Fernsehen, Antenne 2, «Apostrophes», 30. Januar 1987. Bleistiftskizze von Huldrych M. F. Koelbing 
Nach seiner Wiederherstellung beginnt sein dritter Lebensabschnitt, zunächst in Verwirrung und Ratlosigkeit. Während seine Altersgenossen, die sich mit der Besetzung mehr oder weniger arrangiert hatten, ihr Studium abschließen konnten und Stellen in den Spitälern bekleiden, hat er noch nicht einmal die Examen des zweiten Studienjahres abgelegt. Er findet aber auf Umwegen zur Forschung und wird Mitglied einer blendenden Equipe am Pasteur-Institut; André Lwoff und Jacques Monod werden mit ihm den Nobelpreis 1965 teilen.

Wie entgeht der Autobiograph der doppelten Gefahr, sich selbst allzu narzißtisch in den Mittelpunkt zu stellen oder, vielleicht raffiniertere Form der Überheblichkeit, seine eigenen Verdienste ganz auszuklammern? Durch eine möglichst wahrheitsgetreue, in mittlerer Tonlage gehaltene, von Humor aufgehellte Schilderung seiner Befindlichkeiten.

Das Kind ist von den Wörtern angezogen, er dreht und wendet ein Wort, verändert Buchstaben, Silben, läßt sich von Assonanzen leiten, bis ein reines Klanggebilde zurückbleibt. Das gleiche Verfahren, auf den Namen «de Gaulle» angewendet, ergibt Gaule, Goal, Goth, Gotha, gothique, Gaullegotha, Gaullegothique. Und tatsächlich findet er etwas gotisches, etwas kathedralhaftes in der großen Gestalt mit der erhobenen Nase und den Gesten, die Spitzbogen anzudeuten scheinen. Wenn er auch de Gaulle verehrt, dieses Symbol Frankreichs, dem er sich auf Gedeih und Verderb verschrieben hat, schildert er ihn nicht ohne ironische Distanz. Erste Begegnung: Auf dem Schiff, das die frisch in England ausgebildeten Freien Franzosen nach Afrika bringt. Unerwartet taucht der General neben dem jungen Freiwilligen auf. Nach einem Blick auf die Abzeichen fragt er:

- Vous êtes médecin?

- Etudiant en médecine, mon Général.

- Ah! (Pause) Quelle unité?

- Compagnie de marche, mon Général.

- Ah!

Zweite Begegnung: In Brazzaville. Dem General werden die Honoratioren vorgestellt. Der Präsident der Frontkämpferorganisation, ein Stotterer, der angesichts des hohen $\mathrm{G}$ astes mehr noch als sonst ins Stottern gerät, bittet im Namen seiner Kameraden, der General möge ihnen «le plaisir» seines Besuches machen. Darauf de Gaulle: «L'honneur, monsieur, l'honneur. Au suivant.»

Dritte Begegnung: 19 Jahre später, Empfang der Alten Garde im Elysée. 
Der General, weniger schlank, weniger spitzbogig, geht von Gruppe zu Gruppe.

- Ah, Jacob. Content de vous revoir. (Pause.) Que faites-vous maintenant?

- De la recherche scientifique, mon Général.

- Ah! Très intéressant. Dans quel domaine?

- Biologie, mon Général.

- Ah! Très intéressant. Quel genre?

- Génétique, mon Général.

- Ah! Très intéressant. Et où travaillez-vous?

- A l'Institut Pasteur, mon Général.

- Ah! Très intéressant. Vous avez ce qu'il vous faut?

Jacob, nach kurzem Zögern: «Non, mon Général.» Antwort: «Au revoir».

Der Großvater, General auch er, hatte dem Enkel eingeschärft: «Admirer, oui. Idolâtrer, non.» Und so mischt sich in allen Beschreibungen Jacobs neben aufrichtiger Bewunderung häufig eine Spur Spott. Sein Stil ist flüssig, liest sich leicht, eine Frucht jener 10 grauen Jahre, die er im Lycée Carnot verbrachte. Personen skizziert er treffend, oft mit drei Eigenschaften beginnend, etwa: «rond de corps, blanc de teint, gras de peau» (Karl-Heinz); «petit, rondouillard, l'œil rigolard» (Hauptmann C.); «petit, mince, noir de poil» (Oberst B.); «grand et fort, les yeux clairs, le visage ouvert» (H. Marcovich); «grand, élégant, affable» (E.-F. Terroine); «petit, trapu, le nez cassé, l'air d'un boxeur poids plume» (S.Spiegelman); "grand, mince, très droit» (A. Lwoff); «long, déguingandé, débroussaillé» (J. Watson); «petit, replet, le cheveu plutôt rare» (S. Luria); "petit, râblé, l'œil plissé» (J. Weigle); die Liste ließe sich verlängern. Ein bißchen wirkt das Ganze wie angelernt, wie der Aufsatz eines Musterschülers, der die Marotten seines Lehrers kennt und darauf eingeht. Man muß aber zugeben, daß diese knappen Strichzeichnungen ihre Modelle erstaunlich genau einfangen. Jedenfalls erspart uns Jacob jenen rhetorischen, seinerzeit bewunderten, heute fast unerträglichen Stil, den er von André Malraux, einem Vordenker seiner Generation, leicht hätte erlernen können: höchstens da und dort schimmert Pathos durch, etwa im Satz: «Ce que l'homme cherche jusqu'à l'angoisse dans ses dieux, dans son art, dans sa science, c'est la signification.»

In der Schilderung seiner Forschungsarbeit begnügt sich Jacob mit kurzen Angaben, und der Laie muß sehen, was er sich vorstellen mag unter Bakterienkolonien, trüben oder klaren Lysehöfen, oder unter den «boîtes», die täglich beimpft werden müssen. Aber das ist alles weniger wichtig als die 
Beschwörung der Atmosphäre ununterbrochenen geistigen Austausches, in der die Arbeit vor sich ging. Der Schock, den eine zu Formalismus und Verknöcherung neigende europäische Wissenschaftstradition durch Kontakt mit der amerikanischen Forschung erfuhr, wird nachfühlbar. Was Europa nach dem Krieg von den USA zu lernen hatte, betraf weniger den technischen Aspekt der Forschung; als weit wichtiger erwies sich der Stil: mehr Freiheit, offenere Kommunikation, weitergehende Gleichberechtigung.

Mehrmals gelingt es dem Autor, höchst verwickelte Vorgänge, zum Beispiel die Kopulation der Bakterien, auch für den Nichtfachmann verständlich darzustellen. Dem Geheimnis der Forschung kommt er aber vielleicht am nächsten, wenn er seinen zweijährigen Sohn Pierre beschreibt, wie er mit einem Löffel spielt, ihn anstößt, noch einmal anstößt, bis er zu Boden fällt, brüllt, damit man den Löffel für ihn aufhebt, ihn aber gleich wieder fallen läßt, wie er die Beschaffenheit der Welt erkundet und die Kräfte, die in ihr wirken, und dabei weint und lacht.

Die Frauen. Zuallererst, tief eingesunken «au plus profond de ma tendresse», die Mutter, an der er alle Frauen messen wird. Tamara, die im Knaben erste dunkle Ahnungen einer vor Kindern versteckten Welt weckt. Beryl, die fünfzehnjährige Amerikanerin, die den Jüngling küßt. Odile, die große, unerfüllte Jugendliebe, die sich einem andern verspricht. Paola, florentinische Sanseverina, die ihn in die körperliche Liebe einweiht. Die französische Prostituierte in London, die vor lauter Begeisterung, einen Freien Franzosen trösten zu dürfen, ihm einen Geldschein in die Hand drückt (fünf Pfund; viel Geld 1940; er kommt sich zunächst wie ein Zuhälter vor, aber in der Umwertung aller Werte, die sein Leben ergriffen hat, paßt das nicht schlecht). Zwei Versuche, mit Frauen zusammenzuleben; bei der ersten hält er es sechs Tage aus, bei der zweiten vier. Und dann Lise («mince, élancée, la taille fine...»), von der er beschließt, daß sie seiner Mutter gleicht, die er heiratet, die ihm vier über alles geliebte Kinder schenken wird - «cette femme, belle, avec ses enfants, superbes, grouillant et piaillant autour d'elle». Kein militanter Feminist, wie man sieht; die Frauen haben einen wohlbestimmten Platz in seinem Leben - aber man kann sich vorstellen, daß sie sich in seiner Nähe wohlfühlen.

Was macht den guten Forscher aus? «Le grand homme, en science, c'est d'abord celui qui sait discerner les bons problèmes au bon moment, quand il y a une chance de leur apporter quelque solution.» Was ist mit denen, die scheitern? Ein Schauspieler, der ausgepfiffen wird, ein Kaufmann, der 
Konkurs macht, das läßt sich nicht vertuschen; aber ein Wissenschafter, der sich verrennt? «Un raté de la science peut longtemps faire illusion.» Einmal auf dem Holzweg, was bleibt ihm anderes als stur weiterzuwursteln? Wie lange kann man auf Ergebnisse warten, bevor man anständigerweise aufgeben sollte? Fünf Jahre? Zehn Jahre? Wohl darf sich der Forscher nicht leicht entmutigen lassen, und er braucht Hartnäckigkeit und Ausdauer, aber auch die Fähigkeit, Vorstellungen fallenzulassen, wenn sie sich als unfruchtbar erweisen. «J'aime les idées fixes, à condition d'en changer.»

Sich selbst gibt Jacob fünf Jahre, und tatsächlich befindet er sich nach fünf Jahren in der Spitzengruppe der aufregendsten Forschung jener Zeit, und nach zehn an der Schwelle des Nobelpreises. Diesen letzten Sieg enthält er uns vor. Er führt uns nur bis zum Punkt, wo alle Elemente beisammen sind, Weihnachten 1960. Das große Abenteuer ist zu Ende, schon regnet die Asche des Vergessens auf die denkwürdigsten Augenblicke. Das war's. War es all das? War das alles? Es bleibt noch ein Abenteuer: Zeugnis abzulegen, das Buch zu schreiben, das heute vor uns liegt.

Und nun hat das Buch einen Übersetzer gefunden, und was für einen großartigen Übersetzer ${ }^{2}$ ! Bei einer Übersetzung, dieser anspruchsvollsten und schlechtest bezahlten aller geistigen Tätigkeiten, kommt es nicht nur auf die Genauigkeit der Einzelheiten an, sondern mehr noch auf die allgemeine Beleuchtung, damit das Werk im anderssprachigen Leser ähnliche Emotionen weckt wie das Original in seinem Sprachkreis geweckt hat. Markus Jakob hat seine Aufgabe hervorragend gelöst, wobei ihm B. MüllerHill bei den wissenschaftlichen Passagen geholfen hat.

Welchen schwierigen Problemen sich der Übersetzer gegenüber sieht, will ich an einem ganz kleinen Beispiel zeigen. Ich habe oben die kurzen Gespräche zwischen Jacob und de Gaulle im Original wiedergegeben. Die reglementarische militärische Anredeform eines Vorgesetzten lauten im Französischen «mon Capitaine, món Général». Obschon die Konstruktion die gleiche ist wie bei «Monsieur», wo man das «mon» weder heraushört noch empfindet, klingt beim militärischen «mon Général» bei aller Achtung und disziplinierten Distanz die Kameradschaft beim Lösen einer gemeinsamen Aufgabe mit. Falls man sich überhaupt ein analoges Gespräch zwischen einem deutschen Sanitäter des letzten Krieges und einem General denken kann, so hätte die Anrede wohl «Herr General» gelautet. «Herr» deutet aber

2 François Jacob, Die innere Haltung. Eine Autobiographie. Aus dem Französischen von Markus Jakob. Zürich, Ammann, 1988. SFr. 44.--. 
nicht die Zusammengehörigkeit, sondern die Kluft zwischen den Klassen der Befehlenden und der Gehorchenden an. Darum wurde diese Anredeform in der Schweizer Armee abgeschafft. Wie nun geht der Übersetzer vor? Er entscheidet sich für «mein Hauptmann, mein General», er nimmt eine undeutsche Wendung in Kauf, um nicht durch eine «korrektere» deutsche die Beleuchtung zu verfälschen. Zwar schrien die Deutschen damals durchaus auch «mein Führer», aber mit solcher Inbrunst und sich überschlagender Begeisterung, daß an eine Parallele zum schlichten «mon Général» der französischen Reglemente nicht zu denken ist. So kann an einem einzigen Wort die ganze heillose Verstrickung zweier großer Völker abgelesen werden.

An einzelnen Stellen muß der Übersetzer das Unübersetzbare einfach stehen lassen, so bei den Wortspielen des Jungen, für die ein deutsches Äquivalent kaum zu erfinden wäre. Eine solche Passage liest sich folgendermaßen: «Anders wieder bei Carmen, von der wir zu Hause eine Schallplatte hatten, bei der ich, sooft ich sie abspielte, der Stelle in der Arie L'amour est enfant de Bohême entgegenfieberte, wo es heißt <... si tu ne m'aimes pas, je t'aime. Et si je t'aime, prengare datoits. Diesen unsinnigen Worten (...) konnte ich nicht widerstehen.» Somit kommt auch der Leser der deutschen Übersetzung nicht ganz ohne elementare Kenntnisse der französischen Sprache aus. Das ist in Ordnung, denn das Buch wendet sich an gebildete Leser. Die Erfolge Umberto Ecos lassen vermuten, daß es mehr gebildete Leser gibt, als ein beliebiger Blick in eine beliebige Illustrierte oder auf ein beliebiges Fernsehprogramm nahelegen würde.

Der Jude Jacob, ein französischer Patriot, durfte sein Leben in die Schanze für seine Heimat werfen und später seine großen Talente zu deren Ruhm und Ehre verwenden. Ein Deutscher gleicher Abstammung wäre entweder vergast worden, oder hätte, nach gelungener Flucht, gegen seine ihn verstoßende Heimat kämpfen müssen. Kann man sich einen deutschen Leser vorstellen, der nicht über diese grauenvolle Selbstverstümmelung seines Volkes nachdenkt?

Prof. Dr. med. Jean Lindenmann

Institut für Immunologie und Virologie

Postfach

CH-8028 Zürich 